\title{
Classification of chest X-ray images using a hybrid deep learning method
}

Panida Songram $^{1}$, Phatthanaphong Chomphuwiset ${ }^{1}$, Khanabhorn Kawattikul ${ }^{2}$, Chatklaw Jareanpon ${ }^{1}$

${ }^{1}$ Polar lab, Department of Computer Science, Faculty of Informatics, Mahasarakham University, Mahasarakham, Thailand

${ }^{2}$ Department of Information Technology, Faculty of Social Technology, Rajamangala University of Technology Tawan-ok, Chonburi, Thailand

\begin{abstract}
Article Info
Article history:

Received Jun 8, 2021

Revised Dec 14, 2021

Accepted Dec 24, 2021

\section{Keywords:}

COVID-19

Deep learning

Hybrid deep learning

$\mathrm{X}$-ray image classification

ABSTRACT

This work presents a technique for classifying X-ray images of the chest (CXR) by applying deep learning-based techniques. The CXR will be classified into three different types, i.e. (i) normal, (ii) COVID-19, and (iii) pneumonia. The classification challenge is raised when the X-ray images of COVID-19 and pneumonia are subtle. The CXR images of the chest are first proceeded to be standardized and to improve the visual contrast of the images. Then, the classification is performed by applying a deep learningbased technique that binds two deep learning network architectures, i.e., convolution neural network (CNN) and long short-term memory (LSTM), to generate a hybrid model for the classification problem. The deep features of the images are extracted by $\mathrm{CNN}$ before the final classification is performed using LSTM. In addition to the hybrid models, this work explores the validity of image pre-processing methods that improve the quality of the images before the classification is performed. The experiments were conducted on a public image dataset. The experimental results demonstrate that the proposed technique provides promising results and is superior to the baseline techniques.
\end{abstract}

This is an open access article under the CC BY-SA license.

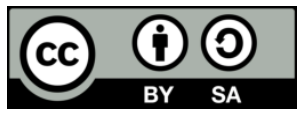

\section{Corresponding Author:}

Chatklaw Jareanpon

Polar lab, Department of Computer Science, Faculty of Informatics, Mahasarakham Universiry

Mahasarakham, Thailand

Email: chatklaw.j@msu.ac.th

\section{INTRODUCTION}

In 2019, the emergence of the Coronaviruses or COVID-19 has undergone and changed people's lives all over the world leading to a global crisis. In April 2021, from world-meter [1], the COVID-19 is affecting 220 countries and territories. Now, the total cases are 150,242,628 cases, separated into 3 groups: i) $3,164,170$ death cases, ii) 127,775,690 recovered cases, and others in the hospital. Many researchers proposed predicting cases of the COVID-19 [2]. Tuli et al. [3] proposed a prediction of the growth and trend of COVID-19 using machine learning (ML) and Robust Weibull model based on iterative weighting. Moreover, from the World Health Organization (WHO) report [4]. The preliminary symptoms of a person who has the COVID-19 include fever, cough, breathing problems, and difficulties. In some cases, the infection can result in pneumonia, severe acute respiratory syndrome, kidney failure, and even death. Unlike normal influenza, the COVID-19 can cause lasting lung damage which takes hold in both lungs [5]. The recovery of it takes time, possibly three months to a year. Ritter et al. [6] proposed a simple statistical model for predicting the level of intensive care load in exponential phases of the disease. The research suggested the model for predicting the ICU rate, which was at $5-18 \%$ with an average of 12 days depending on the area. The long periods of using ICU required special care and treatment from the medical team such as investing, 
disease examination and diagnosis. The main effect in humans appears in lungs displayed in X-radiation (X-ray) images. Conventional analysis of X-ray images can be a workload for doctors and radiologists when there are a huge number of cases. Many researchers tried to develop the research for solving and helping this problem. Pham et al. [7] proposed the survey on artificial intelligence (AI) and big data for COVID-19 pandemic. This paper proposed the overview of AI and big data and identified the application aimed at alleviating against COVID-19, for example, rapid drug strategy, drug discovery, computed tomography (CT) image processing, X-ray reports, and classification using many techniques such as deep learning, case history, and prediction the outbreak. Recently, a number of machine learning and computer vision have been proposed to provide an automated process to classify the X-ray images (CXR) [8]-[19]. Deep learning is one of the favored techniques that are applied. Different deep learning architectures are applied to solve the classification problem of CRX of chest images such as convolution neural networks (CNNs) [8], [10], [11], [14]. Deep learning can generally produce promising results in classifying CRX images, compared to the conventional handcrafted feature techniques [10], [11]. Apart from a single model for the classification, ensemble learning-based is one of the techniques that have been applied for the CRX classification of COVID-19. The stack ensemble technique was introduced to improve the classification results [15]-[17]. However, the ensemble technique can be prone to interpretation issues and computational burden [18]. Zargari el. al. improved the image quality by applying an image normalization technique aiming to standardize the images before the classification was conducted [19]. They demonstrated a marginal improvement over the classification without the image improvement method.

This work proposes a technique for classifying X-ray images of the chest into three types, i.e. (i) normal, (ii) COVID-19, and (iii) pneumonia. The classification challenge is raised when COVID-19 and pneumonia are subtle. In addition, uneven image brightness and poor contrast of the CXR can degrade the images and make the classification more intractable. Therefore, in this work, the images are standardized using a look and feel transfer method. The distribution of color intensity of the images is mapped to one of the predetermined image templates. Then, the contrast of the image will be improved. For the classification, this work applies a deep learning-based technique that binds two deep learning networks, convolution neural network (CNN) and long short-term memory (LSTM), to a hybrid architecture to perform the classification. A deep feature (as an abstract feature) of the images is imposed by a CNN before the final classification is performed using LSTM, which determines the local context of feature vectors). In addition to the hybrid models, this work explores the validity of image pre-processing methods for improving the quality of the images before the classification is performed.

The rest of the paper is organized as follows: Section 2 explains the proposed method of classifying the X-ray images, including proving the details of the baseline methods. Section 3 demonstrates the experiments conducted to evaluate the proposed methods and the results obtained from the experiment. The last section. Section 4, provides a comprehensive discussion of the work before the conclusion is given in this last section.

\section{METHOD}

The objective of this work is to classify the chest X-ray (CXR) images into different chest radiographs mainly focused on some specific diseases and normal chest (e.g., i) normal, ii) COVID-19, and iii) pneumonia). Therefore, this section describes in sufficient detail the proposed method for the classification task. Starting with an overview of the, depicted in Figure 1. through to the different components of the method, which is as follows:

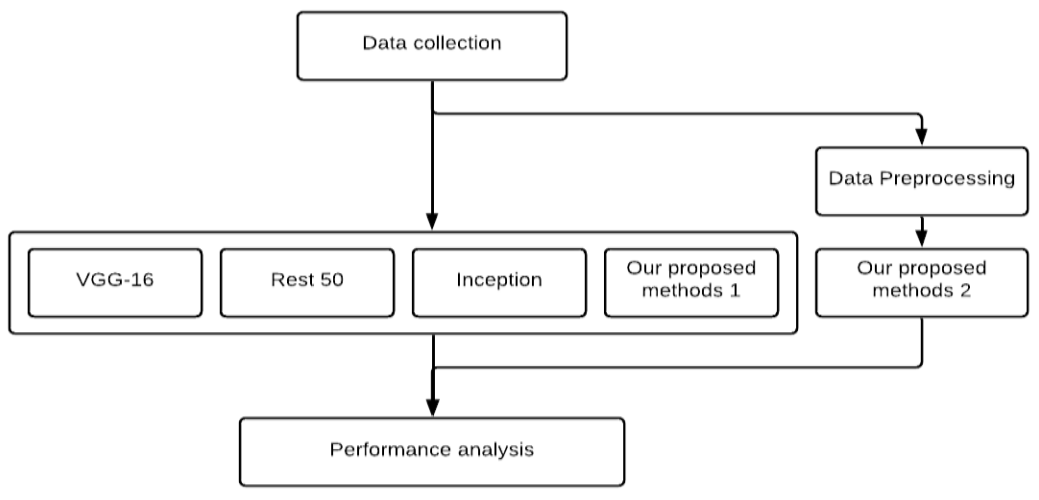

Figure 1. Overview of the work 


\subsection{Data collection}

Our data were collected from a public dataset [20], [21]. The data consisted of 3 classes: i) Normal class ii), COVID-19, and iii) Pneumonia class, which is depicted in Figure 2. The data summarization is demonstrated in Table 1.

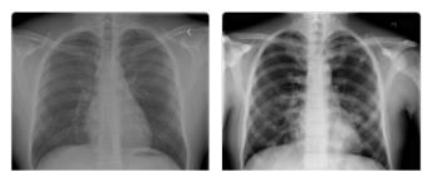

(a)

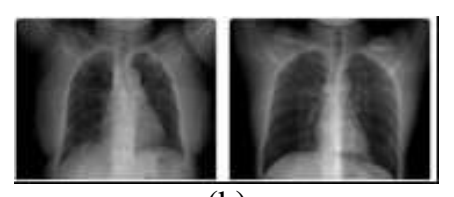

(b)

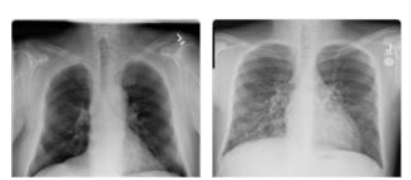

(c)

Figure 2. An example of different radiological types of the X-ray images used in this work: (a) normal, (b) COVID-19, and (c) pneumonia

Table 1. The experimental data

\begin{tabular}{ccccc}
\hline \multirow{2}{*}{ Set } & \multirow{2}{*}{ Total } & \multicolumn{3}{c}{ Target (Class) } \\
& & Normal & COVID-19 & Pneumonia \\
\hline Training & 544 & 184 & 160 & 200 \\
Validate & 136 & 46 & 40 & 50 \\
Test & 150 & 50 & 50 & 50 \\
\hline
\end{tabular}

\subsection{Data pre-processing}

Each of the images proceeds to improve its quality. This work applies two techniques to improve the quality of the images and standardize the data.

- Image standardization: this process is to normalize all images in the data, as to reduce the variation (light and luminance) of the images undergone during the acquiring process. This work applies a technique of the image transformation where the look and feel of the images in the dataset are shifted to the look and feel of a predetermined image, so call a template image $(T)$ [22]. Therefore, the standardized image $\left(I_{S}\right)$ can be obtained by:

$$
I_{s}=\left(\frac{\sigma_{T}}{\sigma_{I}} \times m\right)+\mu_{t}
$$

where,

$$
m=I-\mu_{I}
$$

$\mu_{I}$ and $\mu_{T}$ are the average intensity of pixel values of an input image and the template image. $\sigma_{I}$ and $\sigma_{T}$ are the variance intensity of pixel values of an input image and the template image, accordingly. An example of the standardized images is demonstrated in Figure 3.

- Contrast enhancement: the contrast of the images $\left(I_{c}\right)$ is obtained using contrast limited adaptive histogram equalization (CLAHE) [23]. The input images are standardized before the contrast of the image is enhanced, shown in Figure 4.
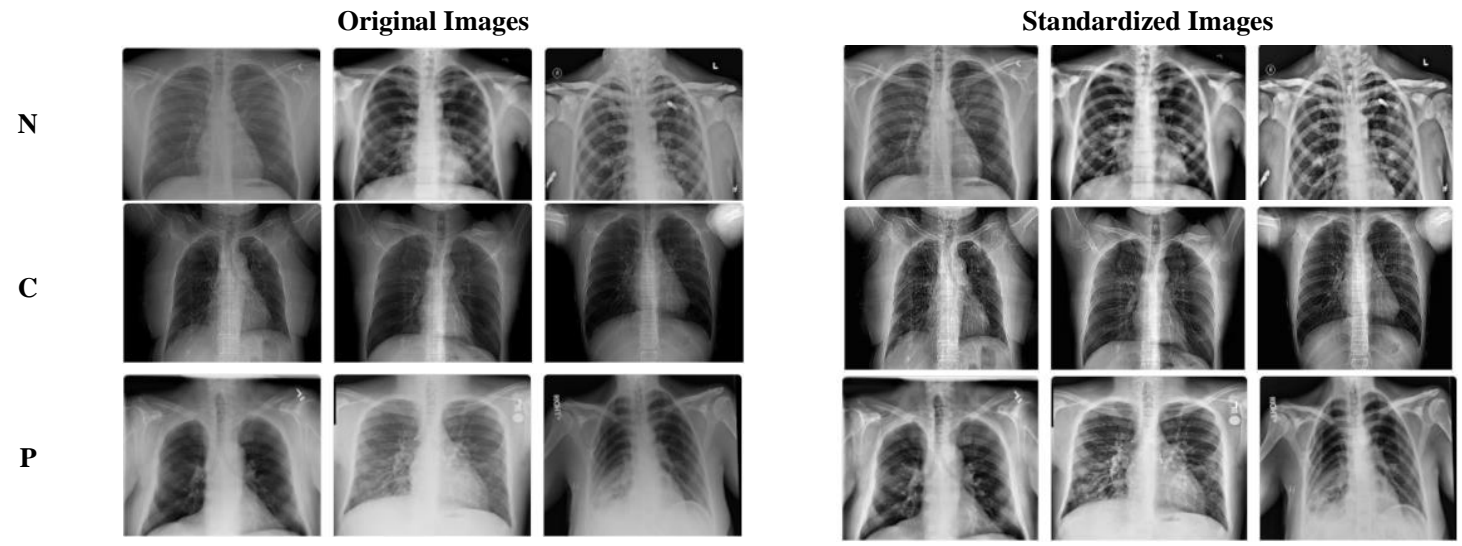

Figure 3. An example of the image standardization using an image transformation technique, 3-leftmost columns are the original image and 3-rightmost columns demonstrate the standardized image of the different image classes 


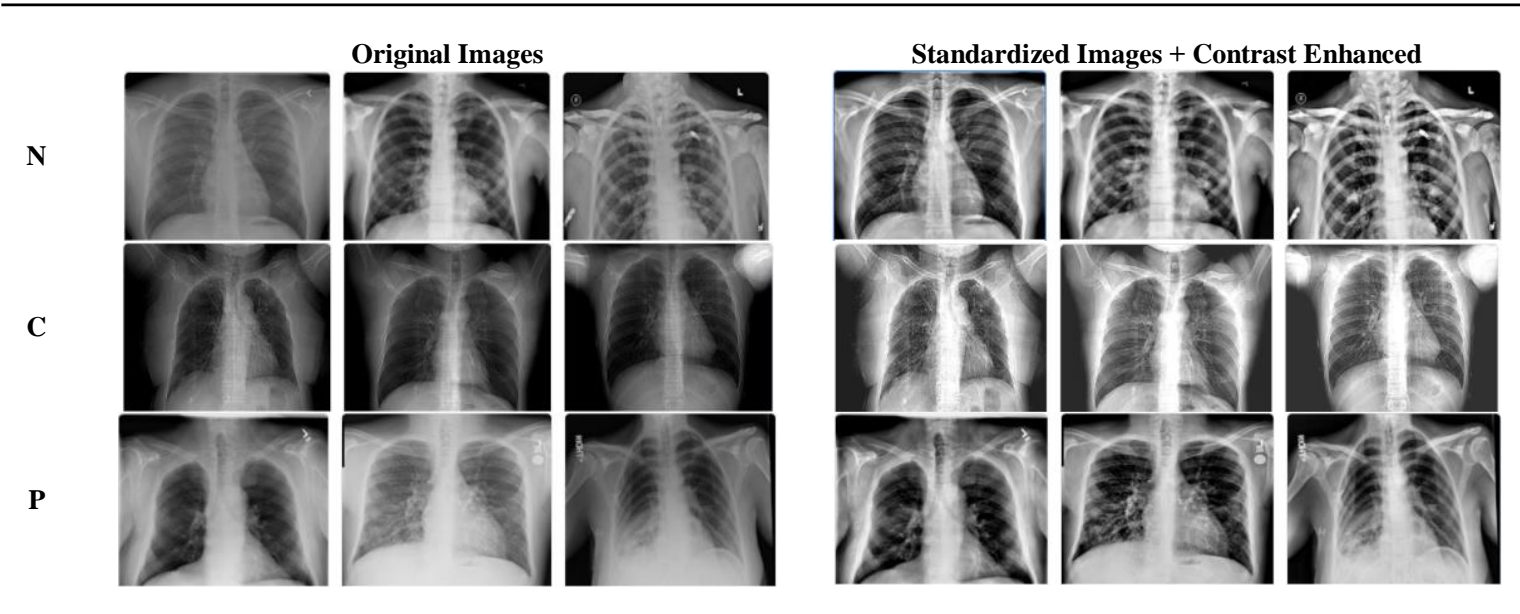

Figure 4. An example of the improved-contrast images, 3-leftmost columns are the original image and 3rightmost columns demonstrate the contrast-enhanced images of the different image classes

\subsection{Deep learning architectures}

In classifying the image data, this work applies deep learning techniques; therefore, this section explains in sufficient detail different deep learning architectures that are implemented. Deep learning architectures are formulated as follows:

$$
I_{l}=\delta_{l}\left(W_{l} * I_{l-1}+b_{l}\right)
$$

where $1 \leq l \leq L$ denotes the layer index and $L$ is the predefined number of layers in networks. $I_{0}$ is an input image, $W_{l}$ and $b_{l}$ are a set of parameters at a layer $l$, * denotes a convolution operator, and $\delta_{l}$ is a layerspecific which is a non-linear function in general. The output of the last layer, $p_{L}$, is input to a softmax function, resulting from a probability value of a given set of the target classes. Then, the classification can be performed by minimizing a loss function with respect to the network weights $(w)$ as (4).

$$
L(f(p), W)
$$

The loss function $(L)$ determines the difference of the prediction obtained by the network $(W)$ and the target of the images. This loss function can be implemented using different techniques, for instance, square loss, logistic loss, exponential loss, and hinge loss. Finally, an optimization technique is performed through training processes to generalize the networks.

- The VGG-16 architecture: The VGG16 convolutional neural network model proposed by Simonyan and Zisserman [24]. The size of the input to the first layer is fixed to $224 \times 224$ pixels. The image is transited through a stack of the convolutional layers, used as the filters. In the stride configuration, a 1-pixel stride is used. Consequently, five max-pooling layers (with stride equal to 2) are used for spatial pooling, performing down-sampling. Max-pooling layers proceed with a 2x2-pixel window. Finally, there are fully connected layers which are three numbers with the channel size as 4096, 4096 and 1000, respectively.

- The ResNet 50 architecture: The residual networks (ResNet) is a type of convolution neural network that is trained with more than 150 neural layers. RestNet was proposed by He et al. in 2015 [25]. The advantage of the ResNet is the simplicity and practicality of usage. It can be applied in many tasks such as detection, segmentation, and identification. The features extracted from the RestNet layer can represent class-specific properties, which can provide promising performance as compared to features extracted from similar network architectures.

- The Inception architecture: The Inception is a deep learning architecture that can aggregate multiple filter sizes. The network was proposed by Christian Szegedy in 2014 [26]. This architecture comprises with of all convolutional layers (ranging from $1 \times 1,3 \times 3$, and $5 \times 5$ ) with output filter backs that are aggregated into a single output vector.

\subsection{Hybrid method}

This section explains the proposed techniques that are used to classify the image data. The proposed architecture relies on 2-sub networks that are consequently connected, as depicted in Figure 5. A CNN is implemented to extract deep features posed in the images. LSTM is deployed as a part of the network to carry 
out the classification. The final layer (1_f) of the CNN is decomposed to 1-D feature vectors before they are fed to LSTM [27]. An optimization is performed in the train process to obtain a generalized model for the classification.

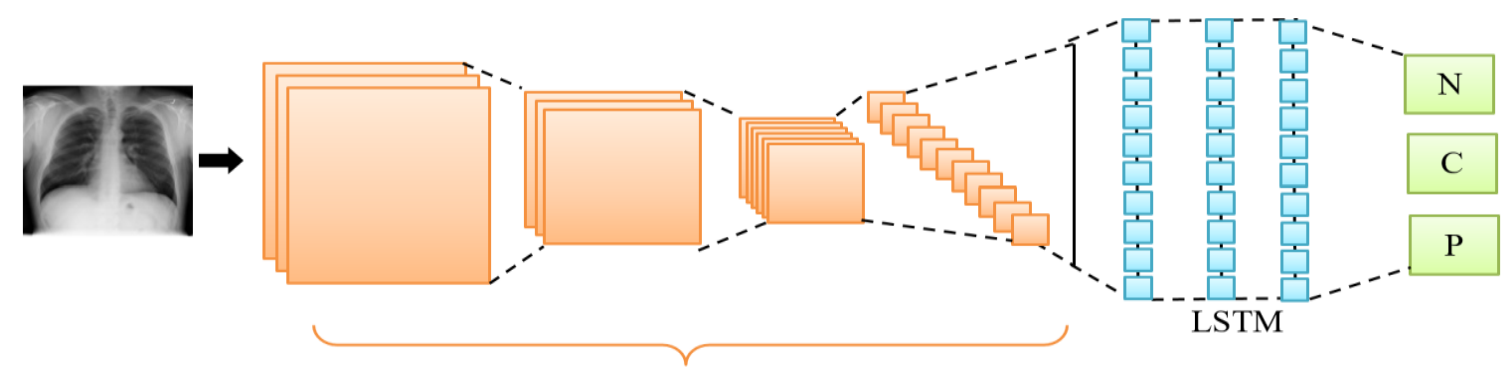

Figure 5. The overall architecture of the proposed method for classifying an input image to the different image types, i.e. i) Normal, ii) COVID-19, and iii) Pneumonia

\section{EXPERIMENTS AND RESULTS}

Section 2 gives the details of the proposed technique. This section, consequently, demonstrates the experiments that were conducted, and the results obtained from the experiment, as to evaluate the performance of the proposed technique. Three deep learning architectures are deployed, i.e. i) VGG-12, ii) RestNet50, and iii) Inception-Net. In the training process, default parameters of each model are set, and the training was carried out using the training data. The test data comprises 150 images of the X-ray from 3 classes of the X-ray types, see section 2.1 for the details. After the experiments were run, the results are demonstrated as the confusion matrix in Tables 2-4.

Table 2. The performance of VGG16

\begin{tabular}{lccccc}
\hline & & Actual class & \multirow{2}{*}{ Precision } \\
\hline Predicted class & Normal & Normal & COVID-19 & Pneumonia & \\
& COVID-19 & 9 & 12 & 9 & 0.63 \\
Recall & Pneumonia & 6 & 30 & 10 & 0.61 \\
& & 0.70 & 0.60 & 31 & 0.70 \\
\hline
\end{tabular}

Table 3. The performance of ResNet50

\begin{tabular}{lccccc}
\hline \multicolumn{5}{c}{ Table 3. The performance of ResNet50 } \\
& & Normal class & COVID-19 & Pneumonia & Precision \\
\hline Predicted class & Normal & 37 & 11 & 14 & 0.60 \\
& COVID-19 & 8 & 28 & 5 & 0.68 \\
Recall & Pneumonia & 5 & 11 & 31 & 0.66 \\
& & 0.77 & 0.56 & 0.62 & \\
\hline
\end{tabular}

Table 4. The performance of Inception

\begin{tabular}{lccccc}
\hline & & Actual class & \multirow{2}{*}{ Precision } \\
& & Normal & COVID-19 & Pneumonia & \\
\hline Predicted class & Normal & 36 & 10 & 10 & 0.64 \\
& COVID-19 & 5 & 32 & 6 & 0.74 \\
Recall & Pneumonia & 9 & 8 & 34 & 0.67 \\
\hline
\end{tabular}

Tables 2-4 shows the results of the baseline techniques in classifying the X-ray images. The accuracy of the baseline techniques is around 65\% of accuracy. The average recall of the baselines is at $66 \%$. RestNet50 provides the lowest recall on the COVID-19 image class, while it can result in promising recall for the normal image class.

In the previous experiment, the baseline techniques for the classification experimented and the results were obtained. Then, the proposals were evaluated. In addition to the original data, in this experiment, 
all the images in the dataset were normalized and enhanced in terms of contrast, using the technique described in Section 2.2. The experiments were conducted, and the results are demonstrated in Tables 5 and 6.

Table 5. The performance of the proposed method without image improvement process

\begin{tabular}{|c|c|c|c|c|c|}
\hline & & \multicolumn{3}{|c|}{ Actual class } & \multirow{2}{*}{ Precision } \\
\hline & & Normal & COVID-19 & Pneumonia & \\
\hline \multirow[t]{3}{*}{ Predicted class } & Normal & 37 & 8 & 9 & 0.69 \\
\hline & COVID-19 & 5 & 31 & 4 & 0.78 \\
\hline & Pneumonia & 8 & 11 & 37 & 0.66 \\
\hline Recall & & 0.74 & 0.62 & 0.74 & \\
\hline
\end{tabular}

Table 6. The performance of the proposed method with image improvement process

\begin{tabular}{lccccc}
\hline & & Normal & Actual class & & \multirow{2}{*}{ COVID-19 } \\
& & Pneumonia & Precision \\
\hline Predicted class & Normal & 40 & 14 & 8 & 0.65 \\
& COVID-19 & 6 & 30 & 4 & 0.75 \\
Recall & Pneumonia & 4 & 6 & 38 & 0.79 \\
\hline
\end{tabular}

The results in Table 5 are obtained from the proposed method. The accuracy of the proposed method is around $71 \%$. The average recall is also $71 \%$. The recall of the proposed technique with the image improvement process gives the best result $(80 \%)$ for the normal class, which positively responds to the results obtained from the baselines. It can also be observed that both the COVID-19 and pneumonia images are usually classified to normal image class when they are miss-classified. The comparison of the baselines and the proposed techniques are summarized in Table 7.

The summarization of the results from the experiment in Table 7 demonstrated that the proposed technique is superior to the baseline technique on the original data (without the image improvement process). In addition, it can be observed that the image improvement process can increase the accuracy of the classification, which results in $72 \%$ of F1-Score.

Table 7. The comparative performance of the 5 methods

\begin{tabular}{lcccc}
\hline & Accuracy & Recall & Precision & F1 \\
\hline VGG16 & 0.64 & 0.64 & 0.65 & 0.65 \\
ResNet50 & 0.64 & 0.64 & 0.65 & 0.64 \\
Inception & 0.68 & 0.68 & 0.68 & 0.68 \\
Proposed method & 0.70 & 0.70 & 0.71 & 0.70 \\
Proposed method + image improvement & $\mathbf{0 . 7 2}$ & $\mathbf{0 . 7 2}$ & $\mathbf{0 . 7 3}$ & $\mathbf{0 . 7 2}$ \\
\hline
\end{tabular}

\section{DISCUSSION AND CONCLUSION}

Chest X-ray images are radiological resources that can be used to determine the severity of respiratory diseases such as COVID-19 and pneumonia. To increase the reproducibility for the disease diagnosis, this proposes a classification technique that separates the X-ray images into different types (COVID-19, pneumonia, and normal). Deep learning-based techniques are used to perform the classification task. The baselines techniques are implemented, comprising VGG-16, RestNet50, and Inception. Then, the proposed technique is constructed. The technique combines two deep neural networks, which are CNN and LSTM. The CNN extracts abstract t-discriminative features from the image and the LSTM oversees the classification process, which can extract the local context of the features generated by the CNN.

The experiment results conducted using a standard dataset show that the proposed technique using the hybrid network architecture yields the best results. In addition, it can be observed that the recall value of the images in the normal class is marginally good, for all classification techniques, compared to the other classes. The X-ray image of the normal class does not contain massively the white matter in the lung area. The image improvement process (both standardization and contrast improvement) can be the key to helping the classifiers to differentiate between the image class. However, considering the COVID-19 and pneumonia image class, the majority of missed classification is found when they are both classified to the normal image class. Therefore, there is a subtle difference between the normal image class the COVID-19 and the pneumonia image class. In addition, it can be remarked from the experimental results that there is a certain level of difference between the COVID-19 image class and the pneumonia class. 
The future work will be aimed at investigating the extension of the proposed technique using the hybrid method. 2D input data for LSTM will also be implemented in future work. In addition, ensemble techniques that combine different deep learning architecture will be investigated in the future work.

\section{ACKNOWLEDGEMENTS}

This research project was financially supported by Faculty of Informatics, Mahasarakham University, Thailand.

\section{REFERENCES}

[1] Worldometers. (2021, April 28). Active cases, [Online]. Available: https://www.worldometers.info/coronavirus/?utm_campaign= homeAdvegas 1?\%22, Access 29 April 2021.

[2] J. Laguarta, F. Hueto, and B. Subirana, "COVID-19 Artificial Intelligence Diagnosis Using Only Cough Recordings," IEEE Open Journal of Engineering in Medicine and Biology., vol. 1, pp. 275-281, Sep. 2020, doi: 10.1109/OJEMB.2020.3026928.

[3] S. Tuli, R. Tuli, and S. S. Gill, "Predicting the Growth and Trend of COVID-19 Pandemic using Machine Learning and Cloud Computing," Internet of Things., vol.11, Sep. 2020, 100222, doi: 10.1016/j.iot.2020.100222.

[4] WHO, COVID-19 Essential Supplies Forecasting Tool: Overview of the structure, methodology, and assumtions used, Interim gyudance, 25 August 2020.

[5] P. Galiatsatos. COVID-19 Lung Damage. [Online]. Available: https://www.hopkinsmedicine.org/health/conditions-anddiseases/coronavirus/what-coronavirus-does-to-the-lungs (accessed Apr. 22, 2021).

[6] M. Ritter, D. V. M. Ott, and F. Paul, "COVID-19: a simple statistical model for predicting intensive care unit load in exponential phases of the disease," Sci Rep., vol. 11, no. 1, Mar. 2021, doi: 10.1038/s41598-021-83853-2.

[7] Q. Pham, D. C. Nguyen, T. Huynh-The, W. Hwang, and P. N. Pathirana, "Artificial Intelligence (AI) and Big Data for Coronavirus (COVID-19) Pandemic: A Survey on the State-of-the-Arts,” IEEE Access., vol. 8, pp. 130820-130839, Jul. 2020, doi: 10.1109/ACCESS.2020.3009328.

[8] T. D. Pham, "Classification of COVID-19 chest X-rays with deep learning: new models or fine tuning?," Health Inf Sci Syst., vol. 9, no. 2, Nov. 2021, doi: 10.1007/s13755-020-00135-3.

[9] Q. Guan, and Y. Huang, "Multi-label chest X-ray image classification via category-wise residual attention learning," Pattern Recognition Letters., vol. 130, pp. 259-266, Feb. 2020, doi: 10.1016/j.patrec.2018.10.027.

[10] Y. X. Tang, Y. B. Tang, and Y. Peng, "Automated abnormality classification of chest radiographs using deep convolutional neural networks," npj Digital Medicine, vol. 3, no. 1, May. 2020, doi: 10.1038/s41746-020-0273-z.

[11] Z. Xue, "Chest X-ray Image View Classification," in IEEE 28th International Symposium on Computer-Based Medical Systems, pp. 66-71, 2015, doi: 10.1109/CBMS.2015.49.

[12] C. Qin, D. Yao, and Y. Shi, "Computer-aided detection in chest radiography based on artificial intelligence: a survey," BioMed Eng OnLine, vol. 17, Aug. 2018, doi: 10.1186/s12938-018-0544-y.

[13] I. Baltruschat, H. Nickisch, M. Grass, T. Knopp, and A. Saalbach, "Comparison of Deep Learning Approaches for Multi-Label Chest X-ray Classification," Sci Rep, vol. 9, Apr. 2019, doi: 10.1038/s41598-019-42294-8.

[14] G. Jia, H. K. Lam, and Y. Xu, "Classification of COVID-19 chest X-ray and CT images using a type of dynamic CNN modification method," Comput Biol Med, vol. 134, Jul. 2021, doi: 10.1016/j.compbiomed.2021.104425.

[15] A. U. Berliana and A. Bustamam, "Implementation of Stacking Ensemble Learning for Classification of COVID-19 using Image Dataset CT Scan and Lung X-ray”, in 3rd International Conference on Information and Communications Technology (ICOIACT), 2020, pp. 148-152, doi: 10.1109/ICOIACT50329.2020.9332112.

[16] P. Shrivastava, A. Singh, S. Agarwal, H. Tekchandani and S. Verma, "Covid detection in CT and X-ray images using Ensemble Learning," in 5th International Conference on Computing Methodologies and Communication(ICCMC), 2021, pp. 1085-1090, doi: 10.1109/ICCMC51019.2021.9418308.

[17] P. Meyer, D. Müller, I. Soto-Rey, and F. Kramer, "COVID-19 Image Segmentation Based on Deep Learning and Ensemble Learning," Stud Health Technol Inform, vol. 281, May. 2021, doi: 10.3233/SHTI210223.

[18] P. Pintelas and I. E. Livieris, "Special Issue on Ensemble Learning and Applications," Algorithms, vol. 13, no. 6, Jun. 2020, doi: $10.3390 / \mathrm{a} 13060140$.

[19] K. A. Zargari, M. Heidari, and S. A. Shariati, "COVID-Classifier: an automated machine learning model to assist in the diagnosis of COVID-19 infection in chest X-ray images," Sci Rep, vol. 11, no. 1, pp. 1-6, May. 2021, doi: 10.1038/s41598-021-88807-2.

[20] M. E. H. Chowdhury et al., "Can AI Help in Screening Viral and COVID-19 Pneumonia?," IEEE Access, vol. 8, pp. 132665-132676, Jul. 2020, doi: 10.1109/ACCESS.2020.3010287.

[21] R. Tawsifur et al., "Exploring the effect of image enhancement techniques on COVID-19 detection using chest X-ray images," Computers in Biology and Medicine, vol. 132, May. 2021, doi: 10.1016/j.compbiomed.2021.104319.

[22] E. M. Reinhard, B. G. Ashikhmin, and P. S. Bruce, "Color Transfer between Images," IEEE Computer Graphics and Applications, vol. 21, pp. 34-41, 2001, doi: 10.1109/38.946629.

[23] K. Zuiderveld, "Contrast Limited Adaptive Histograph Equalization," in Graphic Gems IV, Academic Press Professional, Inc., USA, 1994. Pp. 474-485.

[24] K. Simonyan and A. Zisserman, "Very Deep Convolutional Networks for Large-Scale Image Recognition," in The 3rd IAPR Asian Conference on Pattern Recognition (ACPR), 2015, pp. 730-734, doi: 10.1109/ACPR.2015.7486599.

[25] K. He, X. Zhang, S. Ren, and J. Sun, "Deep Residual Learning for Image Recognition," in IEEE Conference on Computer Vision and Pattern Recognition (CVPR), 2016, pp. 770-778, doi: 10.1109/CVPR.2016.90.

[26] C. Szegedy, V. Vanhoucke, S. Ioffe, J. Shlens, and Z. Wojna, "Rethinking the Inception Architecture for Computer Vision," in IEEE Conference on Computer Vision and Pattern Recognition (CVPR), 2016, pp 2818-2862, doi: 10.1109/CVPR.2016.308.

[27] A. Sherstinsky, "Fundamentals of Recurrent Neural Network (RNN) and Long Short-Term Memory (LSTM) network," Physica D: Nonlinear Phenomena., vol. 404, Mar. 2020, doi: 10.1016/j.physd.2019.132306. 


\section{BIOGRAPHIES OF AUTHORS}

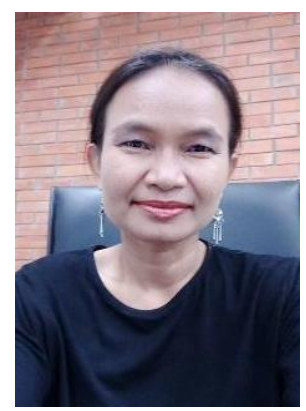

Panida Songram (D) SC SC $\mathrm{P}$ is currently an Assistant Professor at the Faculty of Informatics, Mahasarakham University (MSU), Thailand. She was born in Thailand in 1978. She received the M.Sc. and Ph.D. degree in Computer Science at King Mongkut's Institute of Technology Ladkrabang. Her research areas are association rule mining, sequential pattern mining, text mining, and data mining. In addition, her recent research topics fall into text mining and image processing. For text mining research. She has been attempting to construct a generic learning frame that can extract meaningful information from text, which can be used for different purposes such as using in classification and clustering problems. She can be contacted at email: panida.s@msu.ac.th.

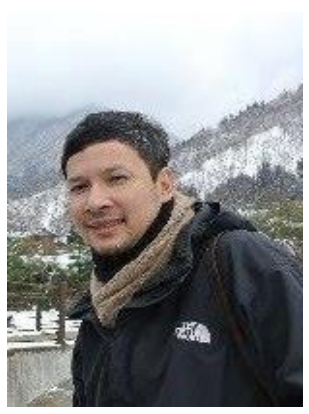

Phatthanaphong Chomphuwiset (iD $\mathrm{SC} P$ is a full-time lecturer at the Department of Computer Science, Mahasarakham University, Thailand. He received a PhD. in computing from the University of Leeds, UK. His main research interests fall into a variety of areas, including medical image processing, Computer Vision applications, and Machine Learning. One of his current research is to investigate techniques in machine learning and deep learning to process histopathological images. He tries to study a computational technique that can cope with the challenge of histopathology image analysis (HIA) where the images pose important challenges such as image size and resolution and the variations of the images - due to the preparation process. He can be contacted at email: phatthanaphong.c@msu.ac.th.

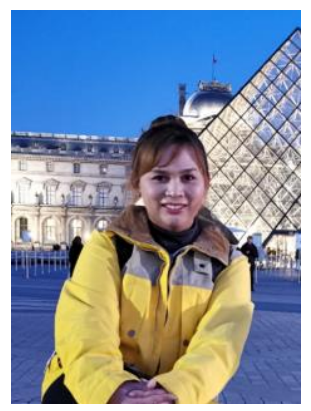

Khanabhorn Kawattikul (D) SI SC P is a full-time lecturer at Department of Information Technology, Faculty of Social Technology, Rajamangala University of Technology Tawanok (Chanthaburi Campus), Thailand. He received a master's degree in information technology from Mahasarakhsm University, Thailand. The main research of interest is Human-Computer Interaction, Computer Vision, and Machine Learning. His current research falls into a wide variety range of computer vision applications, such as image synthetization. $\mathrm{He}$ attempts to generate surreal images of persons from the sketch images. In addition to the information from the image alone, he tries to integrate the information from texts, that are used to explain the visual appearance of the faces. He can be contacted at email: khanabhorn_ka@rmutto.ac.th.

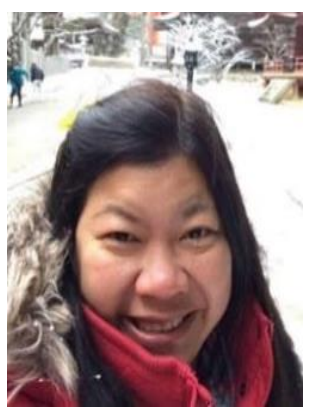

Chatklaw Jareanpon (D) SC P is currently an Assistant Professor of Department of computer science, Faculty of Informatics, Mahasarakham University, THAILAND. Chatklaw received a PhD. in Electrical and Computer Engineering from King Mongkut's University of Technology Thonburi, Thailand. Her interested areas of research are Data Science, AI, Image processing, and Robotics. Currently, she is the head of PoLar Lab at the Faculty of Informatics, Mahasarakham University. She can be contacted at email: chatklaw.j@msu.ac.th. 